\title{
ENSINO DE RETAS E PLANOS COM AUXÍLIO DO SOFTWARE GEOGEBRA 3D MOBILE
}

\author{
TEACHING OF STRAIGHT LINES AND PLANS WITH THE HELP OF \\ GEOGEBRA 3D MOBILE
}

\author{
Murilo Carvalho Feitosa ${ }^{1}$ \\ ORCID iD: $\underline{0000-0003-1163-8838}$
}

Adelmo Artur de Aquino ${ }^{2}$

ORCID iD: $\underline{0000-0002-2119-1825}$

Otávio Paulino Lavor ${ }^{3}$

ORCID iD: 0000-0001-5237-3392

\begin{abstract}
RESUMO
A matemática é aplicada em diversas áreas do conhecimento e o seu entendimento fornece base para aprender outros conteúdos na vida cotidiana e na trajetória acadêmica de qualquer pessoa. No entanto, devido à dificuldade de interpretação e visualização, alguns conceitos, como retas e planos, apresentam índices de aprendizagem não satisfatórios em muitas ocasiões, mas que podem ser contornados com o auxílio de tecnologias nas metodologias de ensino, e com isso vem a ideia da utilização de objetos de aprendizagem. Logo, esta pesquisa tem por objetivo uma investigação prática do ensino de retas e planos auxiliado pelo GeoGebra 3D Mobile. Para tanto, discentes matriculados em turmas de geometria analítica são divididos em dois grupos, sendo um destes de caráter experimental e o outro de controle. $\mathrm{O}$ grupo de controle tem aulas sem o intermédio do recurso tecnológico enquanto que o grupo experimental tem a intervenção do aplicativo em suas aulas. Ao grupo experimental, após apresentação do aplicativo, são ministrados os conteúdos utilizando as funcionalidades da ferramenta em questão. Ao final, todos os alunos são submetidos a uma avaliação de aprendizagem que aponta que os discentes do grupo experimental tiveram resultados mais satisfatórios que o grupo de controle. Isso mostrou que a aprendizagem pode ser dinâmica e interativa quando recursos tecnológicos, como o GeoGebra, são utilizados em sala de aula. A pesquisa revelou apontamentos pedagógicos muito pertinentes quanto a forma com que foram divididos os grupos em questão, trazendo assim contribuições metodológicas consideráveis em pesquisas dessa natureza.
\end{abstract}

Palavras-chave: Ensino de matemática. Geometria analítica. Recursos tecnológicos. Objetos de aprendizagem.

\section{ABSTRACT}

\footnotetext{
${ }^{1}$ Graduando em Engenharia Elétrica pela Universidade Federal Rural do Semi-árido (UFERSA), Mossoró, Rio Grande do Norte, Brasil. Endereço para correspondência: Av. Francisco Mota, 572 - Bairro Costa e Silva, Mossoró - RN, Brasil, CEP: 59.625-900. E-mail: murilocfeitosa@ gmail.com.

${ }^{2}$ Mestrando do Programa de Pós-Graduação em Ensino (PPGE) da Universidade do Estado do Rio Grande do Norte (UERN), Pau dos Ferros, Rio Grande do Norte, Brasil. Endereço para correspondência: BR-405, Km 153, Pau dos Ferros - RN, Brasil, CEP: 59900-000. E-mail: artur-aquino1@ hotmail.com.

${ }^{3}$ Doutorado em Engenharia Elétrica pela Universidade Federal do Rio Grande do Norte (UFRN). Professor Adjunto na Universidade Federal Rural do Semi-árido (UFERSA), Pau dos Ferros, Rio Grande do Norte, Brasil. Endereço para correspondência: BR-226, Km 405, Pau dos Ferros - RN, Brasil, CEP: 59900-000. E-mail: otavio.lavor@ufersa.edu.br.
} 
Mathematics is applied in various areas of knowledge and its understanding provides a basis for learning other content in everyday life and in the academic trajectory of anyone. However, due to the difficulty of interpretation and visualization, some concepts, such as straights and plans, present unsatisfactory learning rates on many occasions, but that can be circumvented with the use of technologies in teaching methodologies, and with that comes an idea of using learning objects. Therefore, this research proposes a practical investigation of the teaching of straights and plans assisted by GeoGebra 3D Mobile. Therefore, students enrolled in analytical geometry classes are divided into two groups, one of which is experimental in nature and the other of control. The control group has classes without the means of technological resource while the experimental group has the intervention of the application in its classes. For the experimental group, after presentation of the application, the contents are taught using the functionalities of the tool in question. In the end, all students are submitted to a learning evaluation that points out that students in the experimental group had more satisfactory results than the control group. This showed that learning can be dynamic and interactive when technological resources, such as GeoGebra, are used in the classroom. The research revealed very pertinent pedagogical notes regarding the way in which the groups in question were divided, thus bringing considerable methodological contributions in researchs of this nature.

Keywords: Analytical geometry. Learning objects. Mathematics teaching. Technological resources.

\section{INTRODUÇÃO}

A matemática é uma das áreas do conhecimento que mais se aplica no nosso dia a dia, nos fenômenos naturais, e em outras incontáveis situações. A apropriação de seu conhecimento de forma aprofundada pode enfrentar algumas dificuldades, em especial na percepção literal em que matemática pode se apresentar, seja na forma de objetos concretos, geometrias, tempo, ou em outras naturezas. Tais dificuldades são evidenciadas quando levamos em consideração o atual cenário educacional do país frente a formação inicial proporcionada pelos cursos de licenciatura, e neste caso àqueles direcionados a formação em matemática. Então, entende-se que as dificuldades de interpretação matemática devem ser superadas para embarcar em situações mais complexas, pois Sepini, Alonso e Maciel (2015) destacam que, para uma melhor sequência didática deve partir das crenças mais simples para as mais complexas.

Passos importantes na construção do conhecimento matemático podem ser dados utilizando a tecnologia como mecanismo auxiliar na apresentação, interpretação e dedução de conceitos. Com isso, Silva (2016, p. 23) afirma,

Acreditamos que a pesquisa contribui para que ações que vinculem os conteúdos matemáticos aos recursos tecnológicos sejam uma prática constante em sala de aula, com fins de proporcionar melhorias na aprendizagem, adequando-se aos recursos presentes nas unidades escolares e incentivando a aquisição de novos recursos uma vez confirmada a necessidade de sua utilização.

Diversas metodologias educacionais dispõem de tecnologias de fácil acesso, tornando um ambiente propício a um ensino diversificado e motivador. Dentre os recursos tecnológicos disponíveis, destacam-se os Objetos de Aprendizagem (OA’s) que são ferramentas digitais 
reutilizáveis e possuem uma proposta interativa e instrucional no processo de ensinoaprendizagem. "Esses objetos podem ser empregados como um instrumento para auxiliar o professor a criar novas estratégias de ensino que possam favorecer a apropriação do conhecimento pelo aluno" (BRAGA et al, 2012, p. 91).

Nesi et al. (2019, p. 564) faz a seguinte constatação acerca da utilização dos OA's:

É possível concluir que os OA podem proporcionar metodologias favoráveis ao ensino e aprendizagem da Matemática, promovendo momentos de investigação, organização e criação de estratégias matemáticas que talvez não ocorram quando se usam apenas os recursos tradicionalmente disponíveis, tais como o quadro-negro, lápis e papel.

Nesse sentido, é necessário que se saiba diferenciar esses recursos digitais que possuem potencial para o ensino, seja este em sua essência um OA ou uma ferramenta computacional que não seja para fins educacionais, mas que com as devidas estratégias, possa-se empregar-lhe o papel de um OA. Ter isso em mente garante que inúmeras ferramentas possam ser utilizadas nas práticas de ensino junto a um planejamento adequado.

Além da arquitetura empregada nos métodos de desenvolvimento, enfatiza-se o reuso desses mecanismos, sendo essa a principal característica que categoriza um recurso digital como sendo um OA (BRAGA et al., 2012).

Levando em consideração a discussão feita até aqui, chega-se a um ponto extremamente significativo da pesquisa, que é a utilização de uma ferramenta digital para o estudo da matemática ocupando o papel de um OA. A atribuição de um plano de atividades sistematicamente bem definido, com objetivos claros e concisos quanto às práticas de ensinoaprendizagem fazem com que esta ferramenta ocupe essa classificação. Inicia-se agora uma discussão acerca das potencialidades de ensino e da aprendizagem de matemática com a utilização do software GeoGebra e suas variações.

O GeoGebra é uma ferramenta que possui recursos de representação e visualização de figuras geométricas. A respeito, Iranzo e Fortuny (2009, p. 442) destacam:

Pudemos verificar neste estudo que a maioria dos estudantes utiliza ferramentas algébricas e de medição e considera que o GeoGebra os ajuda a visualizar o problema e evitar obstáculos algébricos. De maneira geral, os alunos tiveram poucas dificuldades em relação ao uso do software e alguns obstáculos já são obstáculos cognitivos transferidos para o software. O uso do GeoGebra, portanto, promove um pensamento mais geométrico e facilita um suporte visual, algébrico e conceitual para a maioria dos estudantes. Consideramos que o uso do GeoGebra também favorece múltiplas representações de conceitos geométricos, ajuda a evitar obstáculos algébricos, permitindo focar em conceitos geométricos e resolver problemas de outra maneira. 
De acordo com o discorrido, é importante o leitor notar que o GeoGebra traz um suporte apropriado ao professor e ao aluno para que juntos possam construir uma apropriação adequada dos conhecimentos matemáticos estudados.

A geometria analítica, enquanto currículo, carece não somente do estudo de problemas que sejam resolvidos analiticamente, por assim dizer, mas que por sua natureza também necessita de uma visualização mais completa acerca de suas configurações gráficas em planos de duas e três dimensões. Tal disciplina é ofertada na maioria dos cursos de engenharia e podese afirmar que sua compreensão no início do curso fornecerá alicerces para disciplinas mais complexas que requerem conhecimento prévio acerca da geometria.

Em virtude disso, faz-se necessário a pesquisa e a exposição de tópicos tão importantes para o ensino da matemática, buscando versar possibilidades de utilização prática e eficiente em currículos de curta duração, essencialmente para que sejam desenvolvidos em sala de aula. Outro fator que justifica essa pesquisa é a possibilidade de interpretação e visualização de conceitos matemáticos mais complexos, que neste caso refere-se ao estudo das retas e planos. Com isso, acredita-se que a utilização dos OA's possa criar situações de aprendizagem significativa através da motivação desejada.

Nesse sentido, norteamos os problemas da pesquisa quando indagamos: De quais formas os conceitos da geometria analítica, essencialmente sobre planos e retas, podem ser trabalhados em sala pelo professor? E principalmente, em quais perspectivas é possível ensinar essa disciplina frente a utilização de recursos tecnlógicos?

Dessa forma, propõe-se uma atividade de investigação do GeoGebra na disciplina de geometria analítica, visto que o uso de software nesta disciplina alivia as dificuldades de aprendizagem (TENÓRIO; SOUZA; TENÓRIO, 2015). O plano de atividades foi planejado com a finalidade de aferir se o emprego do GeoGebra 3D Mobile cumpre o objetivo de ser uma ferramenta útil no auxílio dos processos de aprendizagem dos alunos. Este aplicativo é encontrado gratuitamente na Play Store para android ou Apple Store para IOS e é oferecido pelo International Geogebra Institute (Instituto Internacional Geogebra). Nesse aplicativo, se pode representar funções, superfícies e outros objetos em 3D e também é possível resolver alguns problemas matemáticos. A interface e descrição se encontra na seção de materiais e métodos. 


\section{RETAS E PLANOS}

Os conteúdos abordados nesta investigação são sobre retas e planos. A seguir, apresentamos os conceitos que foram trabalhados em sala de aula, os quais objetivaram trazer um apanhado geral acerca de conceitos iniciais que julgamos necessários para melhor compreensão de tópicos mais complexos durante a disciplina.

Com o intuito de aproximar os aprendizes da experiência de implementar no GeoGebra Mobile 3D os conteúdos que serão descritos a seguir, essa seção tem por finalidade de apresentar tais conteúdos de forma simples, fazendo um paralelismo com sua aplicabilidade no software para que ao longo das demais seções o leitor esteja mais familiarizado com alguns conceitos, e assim visualizar melhor a resolução de problemas.

\subsection{Retas}

Segundo Winterle (2000, p. 103), dado um ponto $\mathrm{A}\left(x_{1}, y_{1}, z_{1}\right)$ e um vetor não-nulo $\vec{v}=$ $(a, b, c)$, só existe uma reta $r$ que passa por A e tem direção de $\vec{v}$ e um ponto $\mathrm{P}(\mathrm{x}, \mathrm{y}, \mathrm{z})$ pertence a $r$ se, e somente se, o vetor $\overrightarrow{A P}$ é paralelo a $\vec{v}$ como mostrado na Figura 1.

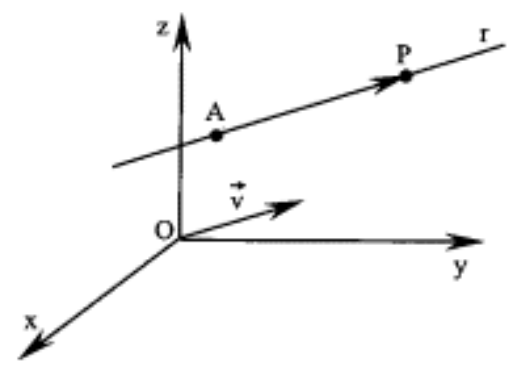

Figura 1 - Representação do ponto $P$, da reta $r$, do ponto $A$ e do vetor diretor $\vec{v}$ Fonte: Winterle (2000, p.103).

Algebricamente, tem-se que

$$
\overrightarrow{A P}=t \vec{v}
$$

para algum número real $t$, chamado de parâmetro.

Da equação (1), obtém-se

$$
P=A+t \vec{v}
$$

que em coordenadas, escrevemos

$$
(x, y, z)=\left(x_{1}, y_{1}, z_{1}\right)+t(a, b, c)
$$

Esta equação é denominada equação vetorial de $r$ e de onde pode-se obter a equação paramétrica da reta, da seguinte forma, 


$$
(x, y, z)=\left(x_{1}+a t, y_{1}+b t, z_{1}+c t\right)
$$

$\mathrm{ou}$,

$$
\left\{\begin{array}{l}
x=x_{1}+a t \\
y=y_{1}+b t \\
z=z_{1}+c t
\end{array}\right.
$$

Para melhor interpretar as configurações as quais as retas podem ocupar, observe a Figura 2 exemplos implementados pelo GeoGebra 3D Mobile.

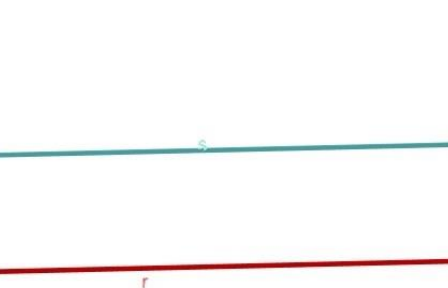

Retas paralelas

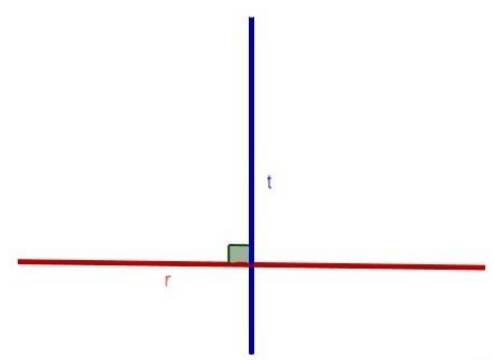

Retas concorrentes perpendiculares

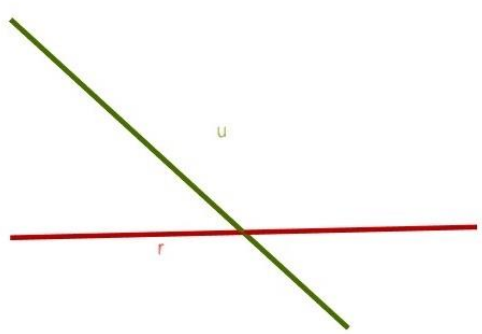

Retas concorrentes não perpendiculares

Figura 2 - Representação de retas em diferentes configurações feitas manualmente no Geogebra 3D Mobile.

Fonte: Autores (2019).

Os exemplos apresentados pela Figura 2 foram retirados do aplicativo Geogebra 3D Mobile, onde inseriu-se equações que representam duas retas paralelas entre si, duas retas concorrentes perpendiculares, isto é, duas retas que possuem um ângulo de $90^{\circ}$ entre si, e por fim duas retas concorrentes não perpendiculares entre si.

A medida com que se altera as configurações, o gráfico é gerado e é passível de interação. $\mathrm{O}$ aprendiz pode testar as diversas posições relativas entre duas retas no espaço, sejam como os exemplos ilustrados, ou também em outras configurações como retas coincidentes e retas não-coplanares. Esse primeiro contato é interessante pois permite visualizar as interações relativas entre retas, mas também a posição de uma reta relativamente a um plano.

\subsection{Planos}

Segundo Winterle (2000, p.125), um plano $\pi$ fica determinado por um ponto $\mathrm{A}\left(x_{1}, y_{1}, z_{1}\right)$ pertencente ao plano e um vetor $\vec{n}=(a, b, c), \vec{n} \neq \overrightarrow{0}$, que é normal ao plano, como pode ser visto na Figura 3. 


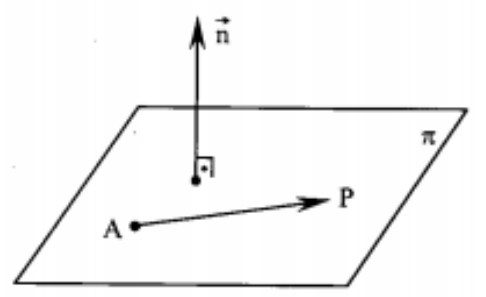

Figura 3 - Representação do plano $\pi$ com o vetor normal, o ponto $A$ e o $P$. Fonte: Winterle (2000, p.125).

Como $\vec{n} \perp \pi, \vec{n}$ é ortogonal a todo vetor representado em $\pi$. Então, um ponto $\mathrm{P}(\mathrm{x}, \mathrm{y}, \mathrm{z})$ pertence a $\pi$ se, e somente se, o vetor $\overrightarrow{A P}$ é ortogonal a $\vec{n}$, isto é,

$$
\vec{n} \cdot(P-A)=0
$$

$\mathrm{ou}$

$$
a\left(x-x_{1}\right)+b\left(y-y_{1}\right)+c\left(z-z_{1}\right)=0
$$

donde temos

$$
a x+b y+c z+d=0
$$

com $d=-a x_{1}-b y_{1}-c z_{1}$, que é a equação geral do plano $\pi$.

Outro aspecto muito importante no estudo dos planos, é o ângulo que se forma entre dois ou mais planos. Para Winterle (2000, p. 136) define-se como ângulo de dois planos $\pi_{1} \mathrm{e} \pi_{2}$ como sendo o menor ângulo formando entre um vetor normal em relação a $\pi_{1}$ com um outro vetor normal a $\pi_{2}$. A Figura 4 a seguir apresentação a configuração característica do cenário descrito.

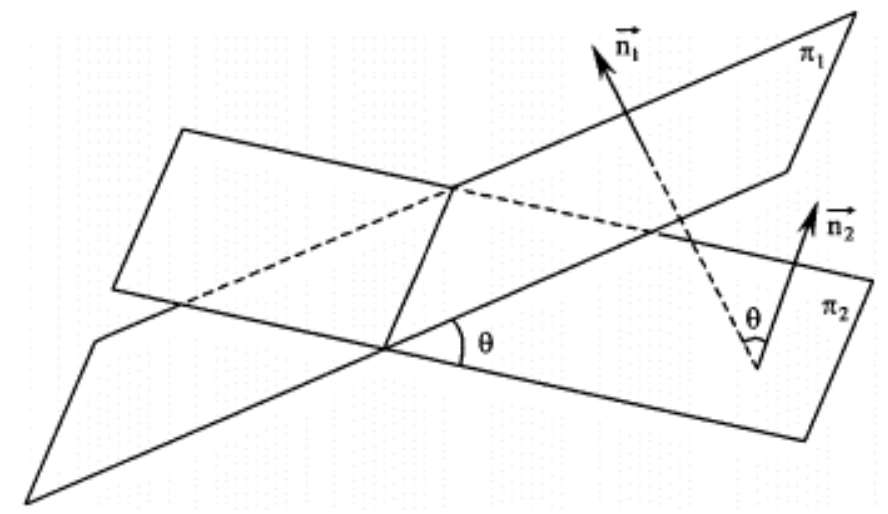

Figura 4 - Representação do ângulo $\theta$ formado entre dois vetores normais à $\pi_{1}$ e a $\pi_{2}$ Fonte: Winterle (2000, p. 136)

Sendo assim, define-se o ângulo $\theta$ como:

$$
\cos \theta=\frac{\left|\vec{n}_{1} \cdot \vec{n}_{2}\right|}{\left|\vec{n}_{1}\right|\left|\vec{n}_{2}\right|}
$$


O ângulo é calculado pelo módulo do produto escalar entre $\vec{n}_{1}$ e $\vec{n}_{2}$, pois este deve ser positivo, dividido pelo produto dos módulos de $\vec{n}_{1}$ e $\vec{n}_{2}$.

Analogamente à seção anterior, analisemos na Figura 5 alguns exemplos de configurações de posições relativas entre planos.

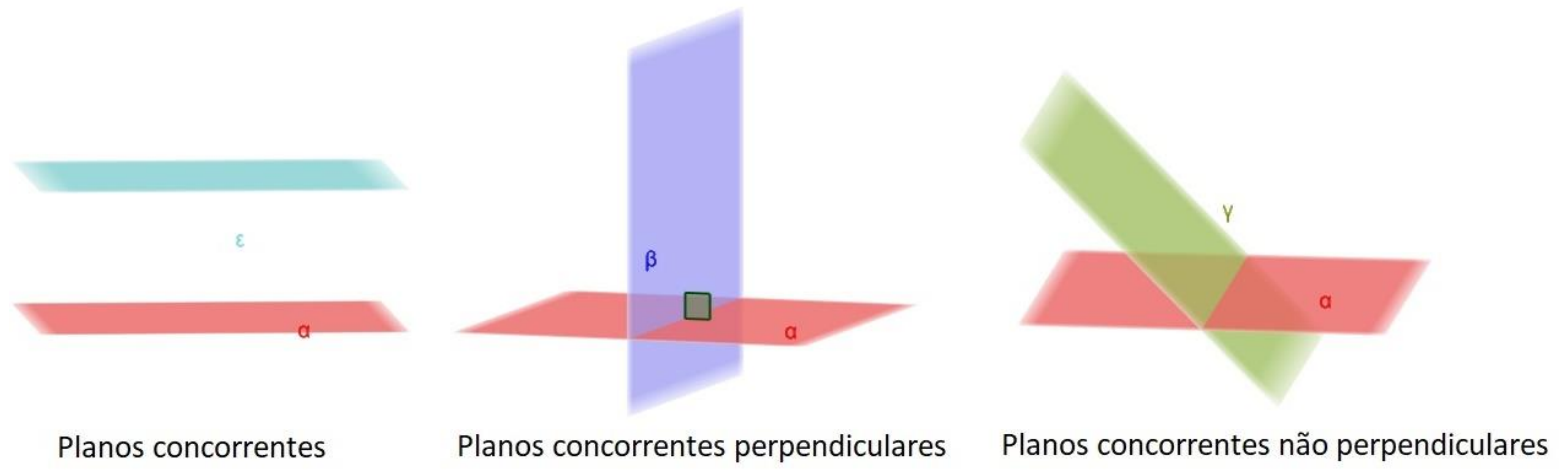

Figura 5 - Representação de planos em diferentes configurações feitas manualmente no Geogebra 3D Mobile.

Fonte: Autores (2019).

Note que os exemplos ilustrados trazem diferentes configurações das posições entre dois planos, que nesse caso são representadas por dois planos concorrentes, dois planos concorrentes e perpendiculares, isto é, que possuem um ângulo de $90^{\circ}$ entre si, e finalmente dois planos concorrentes não perpendiculares.

\section{MATERIAIS E MÉTODOS}

A pesquisa é caracterizada em uma abordagem quantitativa e qualitativa, abordagem na qual é delineada por Rosa, Oliveira e Orey (2015), e visa trazer abordagens que contemplem a elaboração e utilização de currículos de curta duração frente a estratégias de ensino que façam uso de recursos tecnológicos como ferramnetas potencializadoras de aprendizagem, e motivação. O plano de atividades consiste inicialmente no de levantamento e análise bibliográfica contendo estudos teóricos e empíricos acerca da temática, essencialmente àqueles que retratam experiências que fazem uso do software GeoGebra em suas diferentes variações e versões, sejam em plataformas móveis ou para desktop.

Para Trentini e Paim (1999), o estímulo à discussão que concerne às pesquisas de cunho científico no planejamento e elaboração de uma problemática, bem como em sua investigação, possui a revisão bibliográfica como ponto de partida e chegada. Neste caso, os resultados se dão pela estruturação prática de situações avaliadas cuja finalidade é discuti-las e compará-las com os resultados obtidos nesta pesquisa. 
O lócus da pesquisa é caracterizado por discentes matriculados nas turmas de geometria analítica de uma determinada universidade no interior do Rio Grande do Norte, especificamente da cidade de Pau dos Ferros. Estes foram convidados a participar de uma oficina, que durou três dias, cada um com duas aulas de cinquenta e cinco minutos, utilizando o software GeoGebra para apresentação de conteúdos específicos da disciplina em questão. Consoante ao discurso de Oliveira e Chaves (2011), justificamos a escolha pelo GeoGebra 3D Mobile, que se deu por fatores como gratuidade, acessibilidade, manuseio relativamente simples, e principalmente por ser um dos recursos que melhor retrata a representação gráfica de inúmeros conteúdos da matemática, neste caso, especificamente retas e planos.

Em relação a amostragem, trinta e dois discentes dispuseram-se voluntariamente na composição do grupo experimental, em que estes teriam acesso aos conteúdos mediados por tecnologias. Os demais, quarenta e um, tiveram as aulas sem nenhum acesso a objetos de aprendizagem. Este segundo grupo foi denominado grupo de controle. Ao convidar todos os discentes, lhes foram apresentados a proposta da pesquisa de forma clara e objetiva, enfatizando o emprego da metodologia de aulas auxiliadas pelo GeoGebra 3D mobile, com o intuito de facilitar a visualização gráfica e resolução analítica dos conteúdos de retas e planos. Os alunos que formaram o grupo experimental foram os que se disponibilizaram voluntariamente a participar dessa experiência ao ser apresentado o plano de aula. Os demais alunos formaram o grupo de controle.

Esse método de estudo tem sido utilizado por outros autores, como Ruiz e Jiménez (2017) quando buscam os benefícios que o jogo de xadrez traz aos alunos de uma escola estadual de ensino básico e Silva (2016) quando discorre sobre a divisão da sala de aula em dois grupos por faixa etária. No caso desta investigação, os grupos foram selecionados por voluntariedade, como dito. Entendemos aqui que a divisão feita, por não possuir critérios prédefinidos, possa revelar resultados que contribuam na discussão e investigação de variáveis relevantes à prática docente frente ao uso de tecnologias educacionais.

Tendo em vista que todos os discentes do grupo experimental possuem smartphones, escolheu-se a ferramenta em sua versão para plataformas mobile pela flexibilidade proporcionada aos alunos no âmbito da sala de aula. Dessa forma, a aplicação foi feita com auxílio de projetor para que a tela do smartphone do docente fosse projetada para a turma, com o intuito de que todos os discentes pudessem acompanhar as operações realizadas de forma simultânea, possibilitando assim uma maior interatividade durante a aula, uma vez que estes poderiam averiguar os exemplos apresentados de forma paralela a exposição do conteúdo. 
Vale ressaltar que os alunos do grupo experimental instalaram o aplicativo em seus smartphones e previamente destinou-se um tempo para apresentação e funcionamento da interface e funcionalidades do recurso em questão. Esse momento foi empregado a fim de que a aprendizagem dos conteúdos ocorresse de forma mais didática, visto que Silva et al. (2017), afirma que essa forma dinâmica de aprendizado proposta pelo GeoGebra pode favorecer à interação e estreitar os caminhos entre aluno e as novas ferramentas computacionais de aprendizagem.

A Figura 6 mostra a tela inicial do aplicativo GeoGebra 3D mobile.

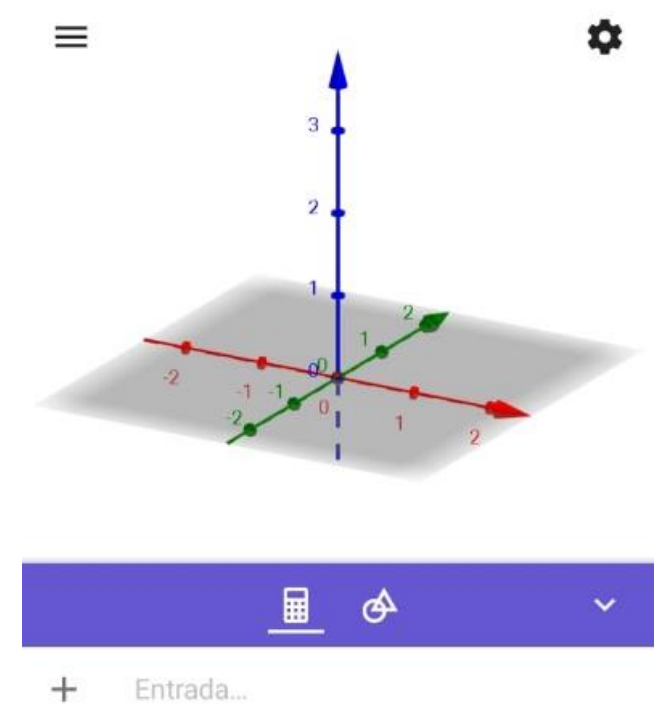

Figura 6 - Tela inicial do GeoGebra 3D mobile Fonte: Autores (2019).

Nesta figura, pode-se ver a tela inicial onde tem-se a representação do sistema tridimensional de coordenadas. É nesta tela que são visualizados os objetos geométricos os quais é possível interagir rotacionando ou alterando os tamanhos do objeto, e que são inseridos na opção de entrada, alocada na parte inferior da tela. Ao clicar nesta opção, surge o teclado e pode-se inserir a função desejada, seja a equação de um plano, uma reta, etc. No canto superior direito observa-se uma engrenagem, onde pode-se modificar as configurações do aplicativo, e no canto superior esquerdo, nas três barras na horizontal, tem-se as opções de excluir, salvar, compartilhar, entre outras.

Segundo Valério e Souza (2013), é importante que após apresentar a ferramenta para os alunos, deve-se apesentar algumas introduções de definições como plano cartesiano, ponto, retas, etc. Então, após a apresentação do aplicativo, a aula foi conduzida com a exposição de 
conteúdos apresentando as figuras geométricas no aplicativo, em que cada operação feita pelo docente é projetada e o estudante pode acompanhar em seu smartphone em tempo real.

Ao final da oficina, foi proposto como instrumento de coleta de dados, um teste para todos os alunos, ou seja, os dois grupos, onde esse teste possui o intuito de aferir se os objetivos de aprendizagem foram alcançados. O grupo experimental foi submetido ainda a um questionário de satisfação.

\section{RESULTADOS E DISCUSSÃO}

Nesta seção, são apresentados os resultados obtidos a partir da prática que foi descrita na seção de materiais e métodos e realizada com o grupo experimental comparando ao grupo de controle. Inicialmente, alguns alunos apresentaram uma certa dificuldade na utilização do aplicativo GeoGebra 3D, entretanto, após a apresentação de sua interface e funcionamento, todos passaram a dominar as funcionalidades de forma plena. Isso facilitou o aprofundamento da exploração dos discentes em relação a utilização do aplicativo, principalmente para melhor visualização dos problemas abordados. Os discentes puderam interagir com a interface do recurso, podendo rotacionar as plotagens, bem como ampliá-las e modifica-las.

Durante as aulas ministradas ao grupo experimental, foi perceptível uma interação e participação efetiva dos educandos enquanto que no grupo de controle, manteve-se uma situação sem apontamentos ou questionamentos por parte dos discentes.

Após as aulas, foi realizado um teste de aprendizagem para os dois grupos, para verificar se os objetivos de aprendizagem foram alcançados, bem como comparar o grupo experimental ao grupo de controle. O teste constou das seguintes questões:

1) Quais as equações reduzidas da reta que passa pelo ponto $\mathrm{A}(-2,1,0)$ e é paralela à reta, $r: \frac{x+1}{1}=\frac{y}{4}=\frac{z}{-1} ?$

2) Determinar o valor de $m$ para que seja de $30^{\circ}$ o ângulo entre os planos: $\pi_{1}: x+m y+$ $2 z-7=0$ e $\pi_{2}: 4 x+5 y+3 z-2=0$.

A Figura 7 mostra a reta e os planos plotados no GeoGebra 3D Mobile. 


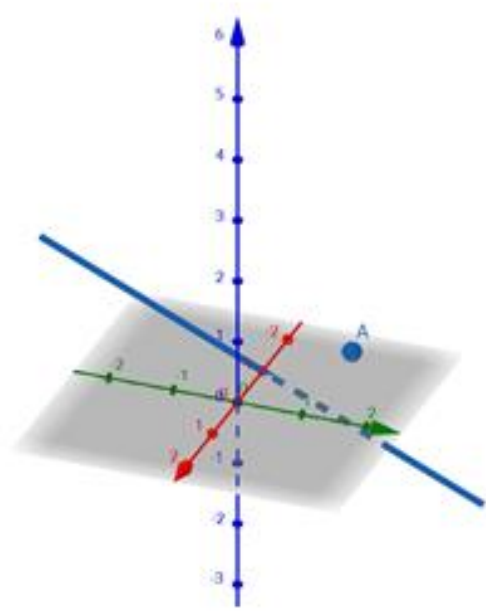

a)

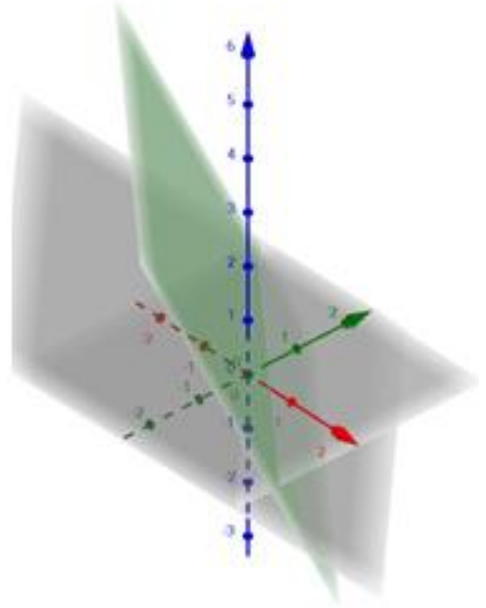

b)

Figura 7 - a) reta e ponto da questão 1 ; b) planos da questão 2 Fonte: Autores (2020).

Fazia parte do teste avaliativo os gráficos plotados na Figura 7, no qual os discentes não faziam uso do aplicativo. O teste avaliativo e o questionário de satisfação foram aplicados separadamente para cada grupo, e em sala de aula, onde os discentes puderam, de forma individual, resolverem os problemas propostos, bem como seus apontamentos pessoais quanto a satisfação de acordo com a sua experiência.

A tabela 1 mostra a quantidade de respostas corretas para as questões 1 e 2 , tanto do grupo experimental quanto do grupo de controle.

Tabela 1 - Resultados do teste

\begin{tabular}{ccccccccc}
\hline & \multicolumn{3}{c}{ GRUPO EXPERIMENTAL } & \multicolumn{3}{c}{ GRUPO CONTROLE } \\
\hline QUESTÕES & $\mathbf{1}$ e 2 & Só 1 & Só 2 & Nenhuma & $\mathbf{1}$ e 2 & Só 1 & Só 2 & Nenhuma \\
\hline $\begin{array}{c}\text { RESOLVERAM } \\
\text { CORRETAMENTE }\end{array}$ & 21 & 6 & 1 & 4 & 9 & 10 & 7 & 15 \\
\hline
\end{tabular}

Fonte: Autores (2019)

Fica evidente que, no grupo experimental aproximadamente $66 \%$ dos envolvidos acertaram totalmente as duas questões, enquanto que, apenas $12,5 \%$ não acertaram nenhuma e $21,5 \%$ acertaram pelo menos uma das duas questões. No grupo controle apenas $22 \%$ acertaram as duas questões, enquanto que 36,6\% não acertaram nenhuma e 41,4\% acertaram pelo menos 
uma. Então, se pode ver que os estudantes do grupo experimental obtivam uma melhor apropriação dos conteúdos de retas e planos previstos em Winterle (2000).

Então, pode-se perceber que há uma diferença considerável, no que se refere ao aprendizado do grupo experimental em relação ao grupo de controle, onde destaca-se que o GeoGebra auxiliando de forma interativa no processo, pode construir um conhecimento matemático mais dinâmico e profundo, corroborando com os resultados obtidos por Oliveira (2016), reforçando seu discurso que retrata o emprego do GeoGebra como ferramenta de apoio ao ensino, onde notou-se em sua pesquisa que os alunos conseguiram obter o aprendizado necessário para o entendimento do conteúdo discutido.

Ao grupo experimental, foi aplicado um teste de satisfação. A tabela 2 mostra os resultados deste teste.

Tabela 2 - Resultados do questionário de satisfação

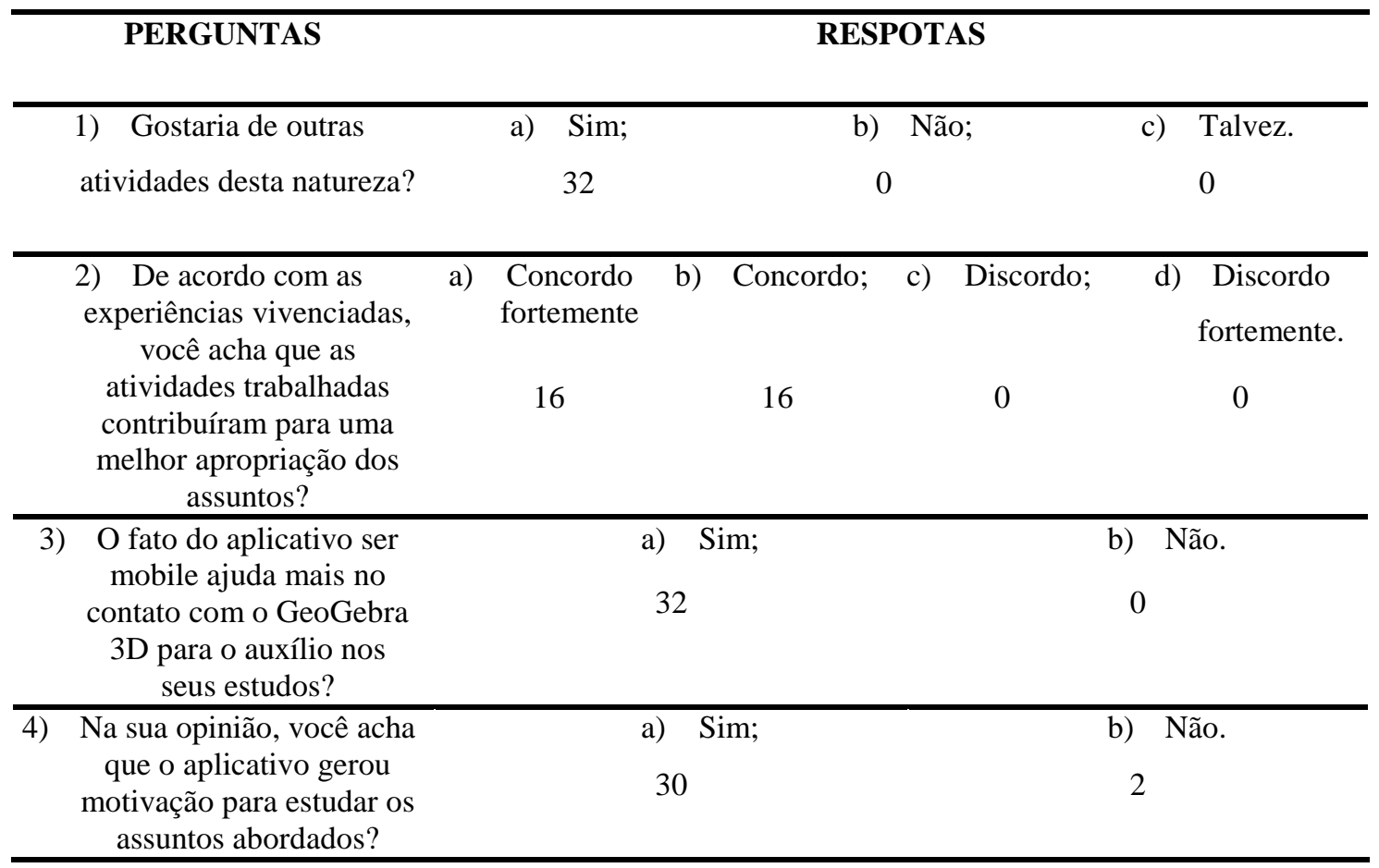

Fonte: Autores (2019)

Veja que os dados da tabela aferem que todos que tiveram a experiência, gostariam de outras atividades desta natureza, bem como concordam que essas atividades lhes ajudaram a obter uma melhor compreensão dos conteúdos. Percebe-se também que o recurso mobile foi bem aceito e isso se dá pelo fato do fácil acesso e manipulação. Quanto a motivação, quase todos afirmaram estar relacionada ao aplicativo. 
O teste de satisfação continha ainda um espaço opcional, onde pedia-se ao aluno que descrevesse a sua opinião sobre o uso dessa metodologia apresentada frente ao uso do aplicativo GeoGebra 3D. Abaixo, no Quadro 1, são apresentados alguns dos comentários.

\begin{tabular}{|c|c|}
\hline \multicolumn{2}{|r|}{ COMENTÁRIOS REALIZADOS PELOS DISCENTES } \\
\hline Discente & Comentário \\
\hline A & $\begin{array}{l}\text { "O aplicativo GeoGebra 3D contribuiu muito no ensino referente ao assunto } \\
\text { abordado, melhorando a visualização do comportamento das retas e planos, } \\
\text { contribuiu muito de uma forma diferenciada, comparada a didática } \\
\text { convencional. Acredito que a didática utilizada serviria para outras disciplinas } \\
\text { como cálculo I, II, etc." }\end{array}$ \\
\hline $\mathrm{B}$ & $\begin{array}{l}\text { "O GeoGebra é um aplicativo altamente versátil e com uma interface simples } \\
\text { de manusear e que é de bastante ajuda nos estudos. E de certa forma, deixa as } \\
\text { coisas mais fáceis para entender o comportamento dos assuntos de geometria } \\
\text { analítica abordados. Creio que possa ser útil em quase todas as disciplinas de } \\
\text { exatas." }\end{array}$ \\
\hline $\mathrm{C}$ & $\begin{array}{l}\text { "A visualização de retas e planos no GeoGebra facilita o entendimento. O } \\
\text { aplicativo mostra de forma clara o comportamento de ambos ao adicionar as } \\
\text { equações." }\end{array}$ \\
\hline $\mathrm{D}$ & $\begin{array}{l}\text { "O aplicativo GeoGebra } 3 \mathrm{D} \text { auxilia no conhecimento por demonstrar de } \\
\text { forma prática os conteúdos vistos na disciplina, trazendo clareza e até } \\
\text { motivação para conhecer a fundo o assunto." }\end{array}$ \\
\hline
\end{tabular}

Quadro 1 - Exemplos de comentários feitos por alunos no questionário de satisfação Fonte: Autores (2019)

Esses foram alguns dos comentários feitos pelos discentes que tinham a liberdade de inserir qualquer comentário acerca da experiência vivenciada. As falas acima mostram o quão significante é a aprendizagem mediada por interações tecnológicas e desperta o interesse para outras atividades em todo o curso.

\section{CONSIDERAÇÕES}

Nesta investigação, conclui-se que muitas dificuldades são observadas em alunos que estão iniciando sua vida acadêmica na área de exatas, dificuldades essas como rotina de estudo, impacto causado pela diferença de realidade para a maioria que vem do ensino público, entre 
outras. Daí, surge a necessidade de uma melhor adaptação de todos os envolvidos no processo de ensino e aprendizagem, para que haja um processo mais dinâmico e motivador nas atividades realizadas em sala de aula. A proposta aqui exposta é de aliar recursos tecnológicos as aulas aproveitando o ambiente a qual se está inserido.

No caso da disciplina de geometria analítica, foi realizada uma atividade de retas e planos com auxílio do GeoGebra 3D na versão Mobile. A fim de verificar a eficácia da proposta, os alunos foram divididos em grupo experimental e de controle. O grupo experimental, o qual tiveram aulas mediadas pelo aplicativo foi participativo e interativo, mostrando resultados mais satisfatórios que o grupo de controle ao serem submetidos a um teste de questões subjetivas.

Consoante a Lucas (2009), os objetivos de bons rendimentos foram observados ao aplicar o teste de verificação de aprendizagem, comprovando a eficácia do uso do GeoGebra 3D mobile no auxílio às aulas de geometria analítica.

A partir desta investigação em que os discentes foram motivados e se apropriaram de conhecimentos de geometria analítica, como foi apontado por eles em seus testes de satisfação e comentários, constatou-se que os recursos tecnológicos são fortes aliados na interação entre professor, aluno e conteúdo. Os resultados obtidos geram uma expectativa para a prática de outras atividades nesta e em outras disciplinas.

Em mãos disto, os resultados deste trabalho foram capazes de validar os objetivos postos em plano. O aplicativo foi capaz de cumprir um papel determinante na aprendizagem dos assuntos abordados, mostrando assim uma perspectiva alternativa no ensino de geometria analítica, essencialmente em práticas que se servem de recursos tecnológicos. Outro ponto positivo revelado, foi a criação de um currículo de atividades de curta duração capaz de adaptar uma ferramenta digital não instrucional, de forma a ocupar o papel de um OA.

Embora os resultados tenham se mostrados satisfatórios, estes também apresentaram características que devem ser apontadas e discutidas. Buscou-se, através dos referenciais utilizados, a elaboração de uma base concisa das variáveis envolvidas na temática em estudo, pois na pesquisa nem sempre as dificuldades se apresentam de forma clara, podendo ser evidenciadas apenas ao longo do estudo a depender dos métodos adotados (MARCONI; LAKATOS, 2003, p. 163).

Mediante isso, acreditamos que a voluntariedade dos participantes a compor o grupo experimental, indica um enviesamento na obtenção dos resultados, uma vez que àqueles que declararam interesse na utilização do aplicativo em suas aulas podem possuir, de forma prévia, alguma experiência com esse tipo de recurso, implicando efeito sob os resultados obtidos. Essa 
dualidade proporciona grandes discussões pedagógicas, pois sendo essa uma possibilidade, precisa-se debater de quais formas pode-se empregar práticas que sejam capazes de contornar a situação de maneira a incluir cada vez mais discentes ao uso dos recursos tecnológicos como ferramentas de apoio em seus estudos.

Tal apontamento destaca-se como uma importante contribuição deste trabalho, visto que muitas pesquisas sequer cogitam possibilidades dessa natureza. Isso significa que, por si só, a tecnologia não é capaz de substituir outras práticas de ensino existentes, acarretando assim uma discussão necessária acerca do conhecimento prévio dos estudantes. Normalmente, muito se discute a respeito dos bons resultados apresentados pelos grupos experimentais, mas deixamos aqui a reflexão relativa as práticas que devem ser tomadas para com aqueles que não possuem pré-disposição a utilização desses recursos, que neste caso retrata a ótico sob o grupo de controle.

A investigação contribui para uma reflexão sobre a prática docente ao considerar objetos de aprendizagem como recursos aliados a motivação, bem como desperta a indagação pelo uso do GeoGebra Mobile como agente facilitador no ensino e aprendizagem de outros conteúdos.

\section{REFERÊNCIAS}

BRAGA, Juliana Cristina; PIMENTEL, Edson; DOTTA, Silvia; STRANSKY, Beatriz. Desafios para o desenvolvimento de objetos de aprendizagem reutilizáveis e de qualidade. In: DESAFIE! 2012, Curitiba.

FEITOSA, Murilo Carvalho; LAVOR, Otávio Paulino. ENSINO DE CIRCUITOS ELÉTRICOS COM AUXÍLIO DE UM SIMULADOR DO PHET. Reamec - Rede Amazônica de Educação em Ciências e Matemática, Pau dos Ferros-RN, v. 8, n. 1, p.126139, 7 fev. 2020. Revista REAMEC. http://dx.doi.org/10.26571/reamec.v8i1.9014. Disponível em: http://periodicoscientificos.ufmt.br/ojs/index.php/reamec/article/view/9014/pdf. Acesso em: 27 mai. 2020.

IRANZO, Nuria; FORTUNY, Josep Maria. La influencia conjunta del uso de GeoGebra y lápiz y papel en la adquisición de competencias del alumnado. Enseñanza de Las Ciencias: Revista De investigación Y Experiencias didácticas, Barcelona, v. 27, n. 3, p.433-446, jun. 2009.

LUCAS, Rodrigo Dantas de. GeoGebra e Moodle no ensino de Geometria Analítica. 2009. 82 f. Dissertação (Mestrado) - Curso de Ensino de Ciências Exatas, Universidade Federal de São Carlos, São Paulo, 2009.

MARCONI, Marina de Andrade; LAKATOS, Eva Maria. Fundamentos de Metodologia Científica. São Paulo: Atlas S.A., 2003. 163 p.

NESI, Taniele Loss et al. Objetos de Aprendizagem de Matemática: um panorama do que diz em alguns estudos no Brasil. RENOTE - Revista Novas Tecnologias na Educação, v. 17, 
n. 1, p. 557-566, 2019. Disponível em:

https://www.seer.ufrgs.br/renote/article/view/96516/54169. Acesso em 19 jan. 2020.

OLIVEIRA, Débora Mary dos Santos. A Modelagem e o Geogebra: sua eficiência e eficácia no ensino da Geometria Espacial. 2016. 21 f. TCC (Graduação) - Curso de Licenciatura em Matemática, Universidade Federal de São João del-Rei, São João del-Rei, 2016.

OLIVEIRA, Elidiane de; CHAVES, Taniamara Vizzotto. A utilização do software GeoGebra como ferramenta de ensino no estudo de retas e ângulos. 2011. Disponível em: http://www.projetos.unijui.edu.br/matematica/cnem/cnem/principal/re/PDF/RE18.pdf. Acesso em: 02 dez. 2019.

RUIZ, Alexandre Antonio de Almeida; JIMÉNEZ, Luís Ortiz. Estudo dos efeitos do jogo do xadrez no ensino da matemática nas turmas de sétimos anos da E.E. Dr. José Carlos Braga de Souza em Itanhaém-SP e suas implicações na aprendizagem. Revista Científica Multidisciplinar Núcleo do Conhecimento, v. 8, n. 4, pp. 81-99, 2019. Disponível em: https://www.eumed.net/rev/atlante/2019/03/jogo-Xadrez-matematica.html. Acesso em 26 mai. 2020.

ROSA, Milton; OLIVEIRA, Davidson Paulo Azevedo de; OREY, Daniel Clark. Delineando e Conduzindo o Método Misto de Pesquisa em Investigações em Educação

Matemática. Perpectivas da Educação Matemática, Ouro Preto, v. 8, n. 18, p. 749-769, 28 dez. 2015. Disponível em: https://periodicos.ufms.br/index.php/pedmat/article/view/828. Acesso em: 26 maio 2020.

SEPINI, Ricardo Pereira; ALONSO, Ángel Vázquez; MACIEL, Maria Delourdes. Análise das mudanças de concepções atitudinais identificados nos estudantes a partir de uma sequência didática com enfoque na natureza da ciência e da tecnologia. Interacções, Cruzeiro do Sul, v. 11, n. 34, p.118-139, maio 2015.

SILVA, Girleide Maria da. Um estudo sobre o uso do GeoGebra na aprendizagem de geometria analítica no ensino médio. 2016. 180 f. Dissertação (Mestrado) - Curso de Profissional em Educação, Universidade Federal de São Carlos, São Paulo, 2016.

SILVA, Rildenir Ribeiro et al. Análise geométrica do estudo das posições relativas entre reta e plano com o Geogebra. Revista Eletrônica de Educação Matemática, Florianópolis, v. 12, n. 1, p.78-86, 2017.

TENÓRIO, André; SOUZA, Sandra Mara Rocha de; TENÓRIO, Thais. O uso do software educativo GeoGebra no ensino de Geometria Analítica. Revista do Instituto Geogebra de São Paulo, São Paulo, v. 4, n. 2, p.103-121, nov. 2015.

TRENTINI, M.; PAIM, L. Pesquisa em Enfermagem. Uma modalidade convergenteassistencial. Florianópolis: Editora da UFSC, 1999. Disponível em:

http://www.scielo.br/scielo.php?script=sci_arttext\&pid=S0034-71671999000400018 Acesso em: 14 fev. 2020.

VALERIO, Alex Aparecido Vaz; SOUZA, Luciane de Fátima Rodrigues de. Ensino da Geometria Analítica com o uso do software GeoGebra. Revista Eletrônica de Educação e Ciência, Avaré, v. 3, n. 1, p.7-14, jan. 2013.

WINTERLE, Paulo. Vetores e Geometria Analítica. São Paulo: Pearson, 2000. 247 p. 
Submetido em: 22 de março de 2020 .

Aprovado em: 25 de maio de 2020. 